\title{
Convivencia escolar: adaptación y validación de un instrumento mexicano en Chile ${ }^{1}$
}

\section{Coexistence in Schools: Adaptation and Validation of a Mexican Instrument in Chile}

\author{
René Valdés Morales (1) revalmorales@gmail.com \\ Verónica López (1) veronica.lopez@pucv.colgmail.com \\ Alicia Alelí Chaparro Caso-López (2) achaparro@uabc.edu.mx \\ (1) Pontificia Universidad Católica de Valparaíso \\ (2) Universidad Autónoma de Baja California \\ (Recibido: 28 de noviembre de 2016; Aceptado para su publicación: 16 de marzo de 2017)
}

Cómo citar: Valdés, R., López, V. y Chaparro, A. A. (2018). Convivencia escolar: adaptación y validación de un instrumento mexicano en Chile. Revista Electrónica de Investigación Educativa, 20(3), 80-91. Recuperado de https://doi.org/10.24320/redie.2018.20.3.1720

\section{Resumen}

El objetivo del presente estudio buscó adaptar y validar en una muestra de 2,868 estudiantes chilenos un cuestionario de convivencia escolar aplicado en México. La adaptación implicó la adecuación lingüística de varios de los ítems del instrumento al contexto chileno, la transformación de la escala de respuesta y la verificación de sus propiedades métricas a través del análisis factorial exploratorio y confirmatorio y de la estimación de la consistencia interna. Los resultados del análisis exploratorio mostraron una estructura factorial semejante a la versión del cuestionario original, además de apropiados niveles de consistencia interna. Una aportación de este estudio fue el análisis factorial confirmatorio con las tres dimensiones integradas en un mismo análisis, los resultados obtenidos sugieren un modelo de segundo orden, en el que la convivencia escolar está formada por prácticas de aula democráticas, inclusivas y de resolución pacífica de conflictos.

Palabras clave: Coexistencia pacífica, secundaria, evaluación.

\section{Abstract}

The objective of this study was to adapt and validate, in a sample of 2,868 Chilean students, a school coexistence questionnaire administered in Mexico. The adaptation involved adjusting the language of several of the items in the instrument to a Chilean context, modifying the answer scale, and verifying its metric properties by conducting an exploratory and confirmatory factor analysis and estimating the internal consistency. The results of the exploratory analysis showed a similar factorial structure to the

\footnotetext{
${ }^{1}$ Este estudio fue financiado por el proyecto FONDECYT 1140960 "Políticas y prácticas de apoyo a la convivencia escolar en ambientes punitivos, segregados y de pruebas estandarizadas con altas consecuencias: el caso de Chile". Agradecimientos a FONDECYT y al proyecto CIE16009 del Programa PIA de CONICYT.
} 
original version, in addition to appropriate levels of internal consistency. One contribution provided by this study was the confirmatory factor analysis with the three dimensions integrated into the same analysis, in which the results suggest a second-order model in which school coexistence consists of practices that are democratic, inclusive, and oriented toward peaceful conflict resolution.

Keywords: Peaceful coexistence, secondary school, assessment.

\section{Introducción}

En el actual escenario educativo en Chile, en marzo del año 2016 entró en vigencia la ley de Inclusión Escolar (Ley No. 20.845). Bajo esta ley se establece, entre otras cosas, que el sistema escolar debe promover y respetar la diversidad, eliminar toda forma de discriminación y asegurar una educación inclusiva de calidad (Diario Oficial de la República de Chile, 2015). Además, si bien las políticas educativas en Chile han asumido el trabajo en convivencia escolar desde la participación de las comunidades escolares (gestión formativa), aún es posible visualizar prácticas más ligadas al castigo y a la sanción (gestión punitiva) para dar respuesta a los diversos problemas de convivencia: disciplinarios, insultos, amenazas, acoso, entre otros (Muñoz, Lucero, Cornejo, Muñoz y Araya, 2014).

Esto plantea enormes desafíos para las escuelas chilenas, puesto que la presencia de la violencia escolar ya sea bajo la forma de bullying u otras formas de discriminación- es una de las barreras más explícitas y reales en las escuelas chilenas, especialmente en aquellas situadas en contexto de exclusión o en riesgo de vulnerabilidad escolar (Carrasco, López y Estay, 2012). Al mismo tiempo, si bien las escuelas son agentes claves en la gestión de la convivencia, éstas tienden a externalizar las atribuciones de responsabilidad (López et al., 2011). Por esto y mucho más, la violencia en las escuelas (no sólo chilenas) es un fenómeno preocupante y de alta incidencia.

Estudios como el realizado por Elijach (2011) para la UNICEF dan cuenta de ello. En este contexto, la convivencia escolar se convierte en un medio y un fin:

La inclusión, la convivencia democrática y cultura de paz, es un medio para mejorar las relaciones humanas, resolver conflictos o prevenir contra la violencia o el fracaso escolar, pero son, sobre todo, un fin primordial de la acción educativa y misión de la escuela. (UNESCO, 2008, p. 17).

A partir de ello, autores como Muñoz et al. (2014) han reconocido que la participación de la comunidad en la generación de una adecuada convivencia resulta clave en la prevención y disminución de los niveles de violencia en la escuela. De esta forma, la convivencia escolar puede ser enseñada, aprendida y "puesta en práctica" en todos los espacios formales e informales de la vida escolar (Sandoval, 2014).

Bajo esta visión, la convivencia escolar puede ser entendida como el conjunto de prácticas de inclusión o de segregación, de participación o subordinación, de solución pacífica o violenta, de las diferencias entre las personas y de las adjetivaciones posibles que adquiere la convivencia -en una escuela singular y en un tiempo determinado (Fierro, 2013; Chaparro, Caso, Fierro y Díaz, 2015). Así, dado el valor y trascendencia de la convivencia escolar, resulta necesario contar con instrumentos de medición que permitan caracterizar este fenómeno de manera rigurosa y con coherencia conceptual (Chaparro, Caso, Díaz y Urías, 2012; Chaparro et al., 2015). La medición apropiada de este constructo contribuiría a establecer las bases para el diseño e implementación de intervenciones específicas y la definición de políticas públicas orientadas a erradicar las diversas expresiones de exclusión, inequidad, falta de participación y violencia, que se observan en el contexto escolar.

\subsection{Definición de la convivencia escolar}

El término de convivencia escolar es polisémico, sus múltiples definiciones complejizan la identificación de los aspectos que la integran. De acuerdo con Fierro et al. (2013), varios enfoques y disciplinas intervienen en su conceptualización: Educación para la paz y derechos humanos, Educación y democracia, Educación inclusiva, Educación y género, Educación y valores, Educación intercultural, Educación cívica y ética, entre otros. La unESCO (2008) propuso la definición de la convivencia escolar, que fue retomada y 
reconfigurada por Fierro, Carbajal y Martínez-Parente (2010), quienes operacionalizaron el constructo de la siguiente manera:

- La convivencia inclusiva se entiende como el reconocimiento a la dignidad de todas las personas partiendo de una valoración de sus características de género, etnia, religión, cultura, grupo social y capacidades, entre otros. Sus ejes son la identidad y cuidado, la valoración de las diferencias y la pluralidad. Destaca la importancia del reconocimiento de logros, esfuerzos y capacidades, el cuidado a las necesidades de otros, el trabajo colaborativo, así como el sentido de pertenencia al grupo de clase y a la comunidad escolar.

- La convivencia democrática refiere a la participación y corresponsabilidad en la generación y seguimiento de los acuerdos que regulan la vida en común, así como el manejo de las diferencias y conflictos. Destaca la importancia de la construcción colectiva de reglamentos y normas con enfoque de principios éticos; así como las decisiones participativas para la acción colectiva, el diálogo reflexivo y el manejo formativo de conflictos.

- La convivencia pacífica que se construye, en gran medida, en función de las anteriores, refiere a la capacidad de establecer interacciones humanas basadas en el aprecio, el respeto y la tolerancia, la prevención y atención de conductas de riesgo, el cuidado de los espacios y bienes colectivos, la reparación del daño y la reinserción comunitaria.

\subsection{Evaluación de la convivencia escolar}

A la fecha, los instrumentos para la evaluación de la convivencia escolar se han centrado principalmente en la identificación de los aspectos negativos de ésta, más que en la exploración de las interrelaciones que promueven la inclusión, democracia y cultura de la paz. Por ejemplo, Ortega y Del Rey (2002) elaboraron un conjunto de instrumentos dirigidos a estudiantes, docentes y familia en los que se recaba información sobre cómo se están abordando los conflictos interpersonales y los problemas de abuso, acoso, prepotencia y maltrato escolar. Peralta (2004), cuyo instrumento se centra en la identificación de problemas de convivencia escolar tales como indisciplina, desinterés académico, conducta antisocial, bullying y comportamiento disruptivo. García (2012), autor de Conves -instrumento dirigido a la identificación y valoración del acoso escolar. Sin embargo, si se tiene en cuenta la consideración de la UNESCO (2008) de que la convivencia escolar es "un medio para mejorar las relaciones humanas, resolver conflictos o prevenir contra la violencia o el fracaso escolar" (p. 17), resultaría entonces necesario contar con un instrumento que más que medir la violencia -como los instrumentos mencionados-, permitiera medir las prácticas que se gestan al interior de la escuela y que promueven una convivencia democrática, inclusiva y pacífica (Fierro et al., 2010).

Bajo esta perspectiva, Chaparro et al. $(2012,2015)$ elaboraron un instrumento de evaluación de la convivencia escolar que retoma las tres dimensiones propuestas por la UNESCO, y que mostró características psicométricas apropiadas en una población de estudiantes de secundaria en México. De este instrumento, Caso et al. (2013) presentaron una versión abreviada, el que también mostró adecuadas características psicométricas y una estructura de tres factores, coherentes con la definición conceptual. Esta versión reducida del instrumento, formada sólo por 31 ítems, proporciona información específica con una descripción clara de las prácticas escolares que promueven condiciones de convivencia democrática, inclusiva y pacífica. Por lo tanto, podría funcionar como una herramienta evaluativa tanto para la generación de información para la mejora, como para establecer parámetros comparativos entre distintos entornos educativos (por ejemplo mexicano y chileno), que ayude a caracterizar las prácticas de convivencia en cada caso.

Sin embargo, es necesario para ello determinar si el instrumento funciona de la misma forma en una población diferente a la que fue desarrollado, para asegurar su uso en estudios comparativos. Con base en ello, el objetivo del presente estudio fue adaptar y validar el Cuestionario de convivencia escolar (Chaparro et al., 2012, 2015; Caso et al., 2013) en una muestra de estudiantes de secundaria de Chile. 


\section{Método}

El estudio involucró a 2,868 estudiantes de 80. año básico de escuelas municipales, particulares subvencionadas y particulares pagadas de tres regiones del país, de los cuales 1,388 son hombres (48.3\%) y 1,444 son mujeres (50.4\%); 36 participantes (1.3\%) no respondieron la encuesta. En la región de Tarapacá participaron 481 estudiantes (16.8\%), en la región de Valparaíso 1,354 (47.2\%) y en la región Metropolitana 1,033 (36\%). La muestra fue representativa de escuelas urbanas de las tres regiones del país. La estrategia de muestreo fue estratificado por dependencia, con un cálculo muestral representativo para cada región con nivel de confianza al 95\% y margen de error del 10\%, conformándose una muestra de 210 escuelas.

Se utilizó el Cuestionario de convivencia escolar en su versión reducida (Caso et al., 2013), instrumento formado por 31 ítems agrupados en tres dimensiones: Inclusión, Democracia y Paz, cuyas propiedades psicométricas de las dimensiones de Inclusión ( $k=12, a=.930)$, Democracia $(k=9, a=.907)$ y Paz $(k=10, a=$ .919), registran correlaciones del orden de .938, .932 y .932 con respecto a las dimensiones del instrumento original de 183 ítems, con un porcentaje de varianza explicada aceptable (45.30\%). Para su aplicación en Chile, se consideró necesario ampliar a cinco niveles las opciones de respuesta (nunca, algunas veces, regularmente, casi siempre y siempre) con el fin de analizar su comportamiento como escala (Carifio y Perla, 2007). El cambio de orden en las opciones de respuesta es sugerente dependiendo de la población encuestada (Abella, Rodríguez, Pérez-Sindín, 2010). En el caso de la muestra de esta investigación, las escuelas ya habían participado de otras investigaciones y aplicaciones de encuestas, donde las categorías de respuestas eran de cinco opciones. Mantener las opciones de respuesta era recomendable para evitar los efectos derivados del sesgo de utilizar un instrumento que carece de punto central (Abella et al, 2010), además es útil mantener la metodología para los estudiantes. Por otro lado, utilizar opciones impares de respuesta funciona mejor en aplicaciones autoadministradas y permite disminuir el error de medición (Asun, 2006).

Como primer punto en el proceso de adaptación, se realizó un análisis léxico y de pertinencia semántica de los ítems con la finalidad de resguardar la precisión lingüística de cada uno a la realidad chilena. No obstante, dado que el instrumento va dirigido a alumnos de enseñanza básica, los ítems no sufrieron grandes modificaciones. Las palabras o frases que fueron modificadas fueron las siguientes:

1. Cada quien: la expresión "cada quien" es poco utilizada en el ámbito escolar y está poco arraigada en el habla cultural de Chile. En su reemplazo se utilizará "cada uno".

2. Padres de familia: prácticamente no se usa en el ámbito escolar el concepto "padres de familia". Para referirse a los tutores, se usa en Chile "padres y apoderados".

3. Platicar: la expresión "platicar" casi no es utilizada, a diferencia de "conversar" o "dialogar", que son término cotidianos.

4. Salón de clases: en Chile es altamente usado el concepto "sala de clases" para referirse al espacio donde generalmente se desenvuelven los estudiantes y se aplica el currículum escolar.

El instrumento fue aplicado en el establecimiento escolar durante la jornada escolar, en forma grupal. Se solicitó que sólo estuvieran presentes aquellos alumnos cuyos padres habían firmado el consentimiento informado. Los docentes no estuvieron presentes durante la aplicación. Para resguardar los aspectos éticos de la investigación, se enviaron cartas de consentimiento informado a padres y apoderados, y se solicitó el asentimiento informado de los estudiantes.

Se realizaron análisis factoriales exploratorios por cada dimensión usando el método de extracción máxima verosimilitud, método altamente recomendado para explicar de mejor manera la varianza observada, pues permite un mejor contraste del ajuste del modelo y se sugiere para instrumentos con cinco o más opciones de respuesta (Lloret-Segura, Ferreres-Traver, Hernández-Baeza y Tomás-Marco, 2014). Se utilizó el mismo método de extracción para el conjunto de ítems con rotación Oblimin. Se realizaron confirmatorios para cada una de las tres dimensiones del instrumento, a fin de verificar la 
estructura del constructo del instrumento mexicano. Además, dado que las tres dimensiones -se asumemiden un mismo constructo, se realizó un Análisis Factorial Confirmatorio (AFC) de segundo orden. Conviene mencionar que el uso de Análisis Factorial Exploratorio (AFE) y AFC no son excluyentes entre sí y pueden usarse en un mismo estudio (Méndez y Rondón, 2012). El AFE permite anticiparse a la búsqueda teórica que implica el AFC, el cual era encontrar un factor mayor y subyacente al instrumento. Por otro lado, como el objetivo de este estudio es adaptar el instrumento mexicano, el uso de ambos métodos permite transparentar el diseño de la investigación en función de los resultados esperados. El AFE aporta al AFC la precisión de las dimensiones subyacentes y es recomendable en estudios de adaptación de pruebas (Mavrou, 2015). Finalmente, dado el alto tamaño muestral de esta investigación, se recomienda aplicar AfE con el propósito de disminuir la probabilidad de error y aumentar la precisión de los estimadores poblacionales (Mavrou, 2015). Junto con lo anterior, se verificó la consistencia interna de las dimensiones a través del alfa de Cronbach. Para los análisis se utilizó el paquete estadístico sPSs 2.1 y el módulo AMOS.

\section{Resultados}

Se presentan a continuación, los resultados de los análisis por cada dimensión de la convivencia.

\subsection{Convivencia inclusiva}

Al realizar el AFE mediante análisis de máxima verosimilitud para la dimensión de convivencia inclusiva y habiendo comprobado los supuestos base $(\mathrm{KMO}=.931$; Bartlett $=11495,2 ; p<.00)$, los resultados indican la unidimensionalidad de la dimensión, la que explica el $43.4 \%$ de la varianza con un alfa de .88. Se observa que todos poseen una carga factorial por sobre .51, lo que es adecuado para la robustez de la dimensión. Sin embargo, la comunalidad del ítem 02 (En mi escuela se atiende a los padres y apoderados que solicitan atención especial para sus hijos) y del ítem 10 (En mi escuela hay personas capacitadas para apoyar a estudiantes con bajo rendimiento) de esta dimensión son bajo .30, lo que si bien no implica eliminarlo -dado su valor límite y la importancia del reactivo- es menor en comparación al resto de los ítems. El resto de los ítems presenta comunalidades entre .31 y .49.

El AFC mostró cargas factoriales aceptables y, en general, índices de bondad de ajuste apropiados, comprobando la estructura unifactorial de la dimensión. No se presentan covarianzas entre estos ítems del instrumento (figura 1). 


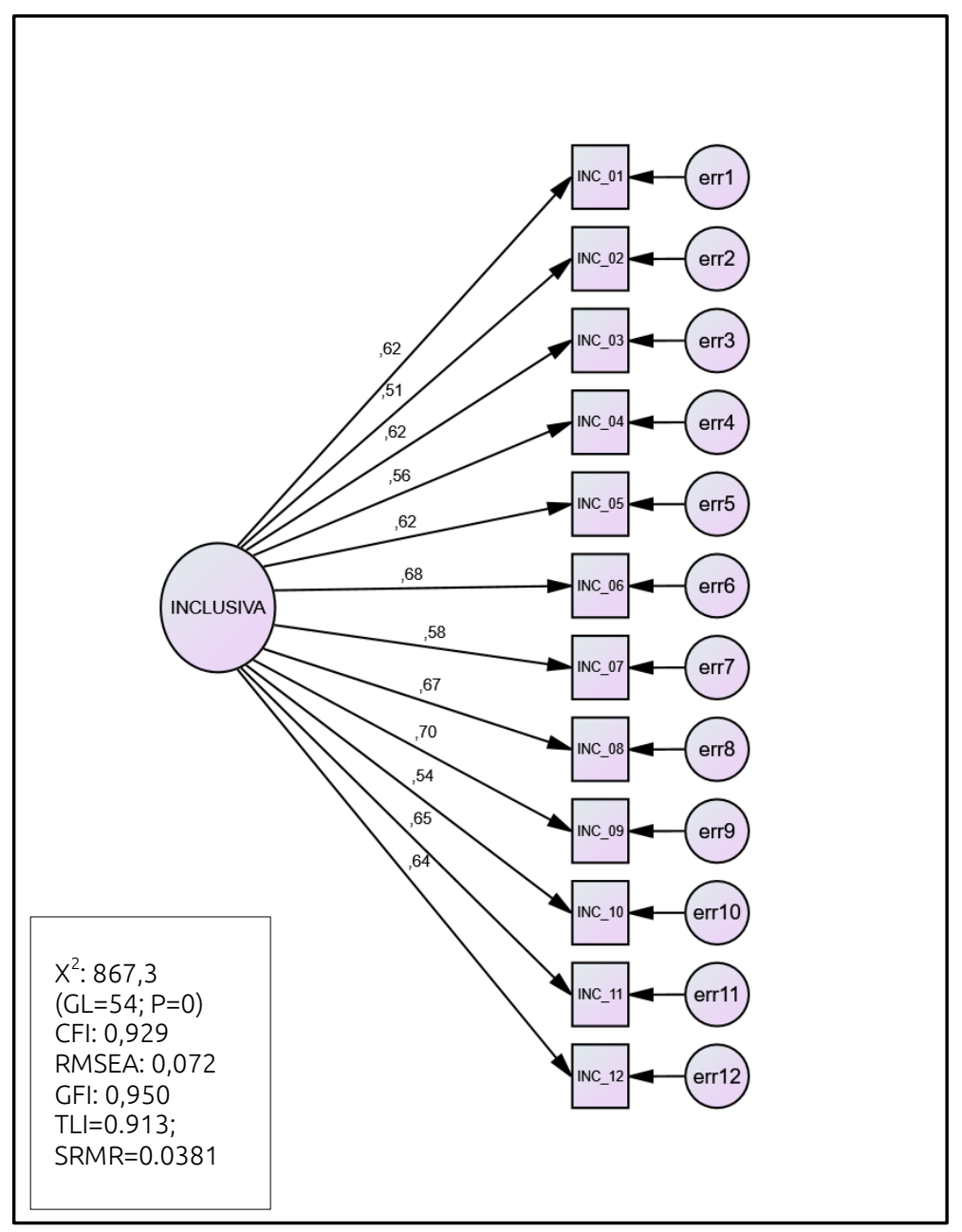

Figura 1. AFC para la dimensión Convivencia inclusiva

\subsection{Convivencia democrática}

Cumpliendo los criterios de factorización ( $\mathrm{KMO}=.925$; Bartlett $=9460.43 ; p<.00)$ y realizado el análisis factorial mediante máxima verosimilitud, los resultados indican la unidimensionalidad de la dimensión Convivencia democrática, el que explica el $50 \%$ de la varianza con un alfa de .87. En el caso de los ítems, se observa que todos poseen una carga factorial por sobre .57 y las comunalidades oscilan entre .32 y .56, lo cual indica que la solución de una dimensión es adecuada.

El AFC para la dimensión Convivencia democrática mostró también cargas factoriales apropiadas y adecuados índices de ajuste comprobándose la estructura unifactorial. Al igual que la dimensión anterior, tampoco se presentan covarianzas entre estos ítems del instrumento (figura 2). 


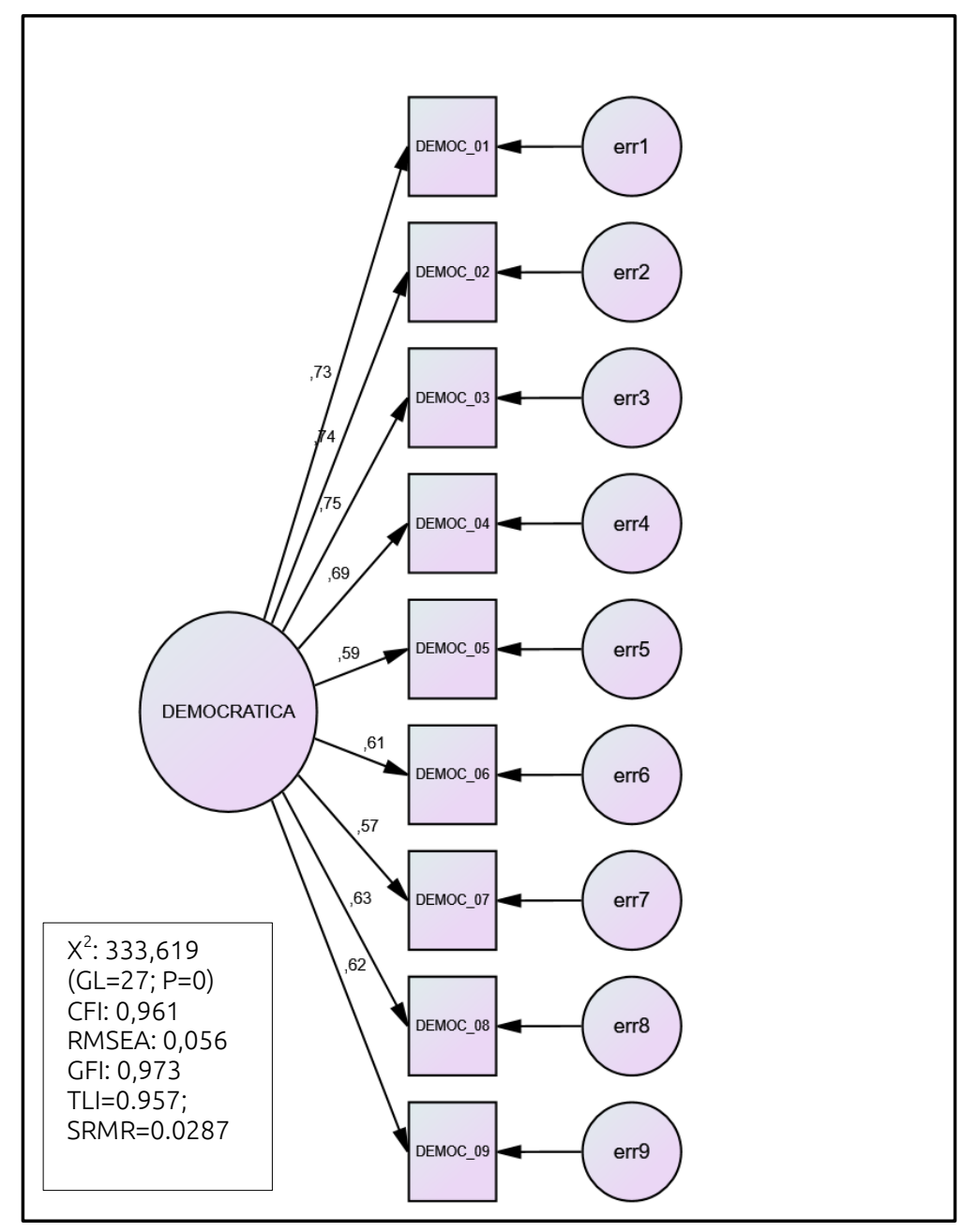

Figura 2. AFC para la dimensión Convivencia democrática

\subsection{Convivencia pacífica}

Para la dimensión Convivencia pacífica el AFE mediante análisis de máxima verosimilitud, y habiendo comprobado los supuestos base $(\mathrm{KMO}=.941$; Bartlett $=14485.50 ; p<.00)$; los resultados indican la unidimensionalidad de los ítems que integran la dimensión, el que explica el 55.7\% de la varianza con un alfa de .910. En el caso de los ítems, se observa que todos poseen una carga factorial por sobre .61 y las comunalidades oscilan entre .37 y .66, lo cual indica que la solución de una dimensión es adecuada.

En cuanto al AFC realizado para la dimensión Convivencia pacífica, igual que en los casos anteriores éste mostró adecuados índices de ajuste, con cargas factoriales aceptables, comprobando así la estructura unifactorial de la dimensión. Al igual que las dos dimensiones anteriores, tampoco se presentan covarianzas entre estos ítems del instrumento (ver figura 3). 


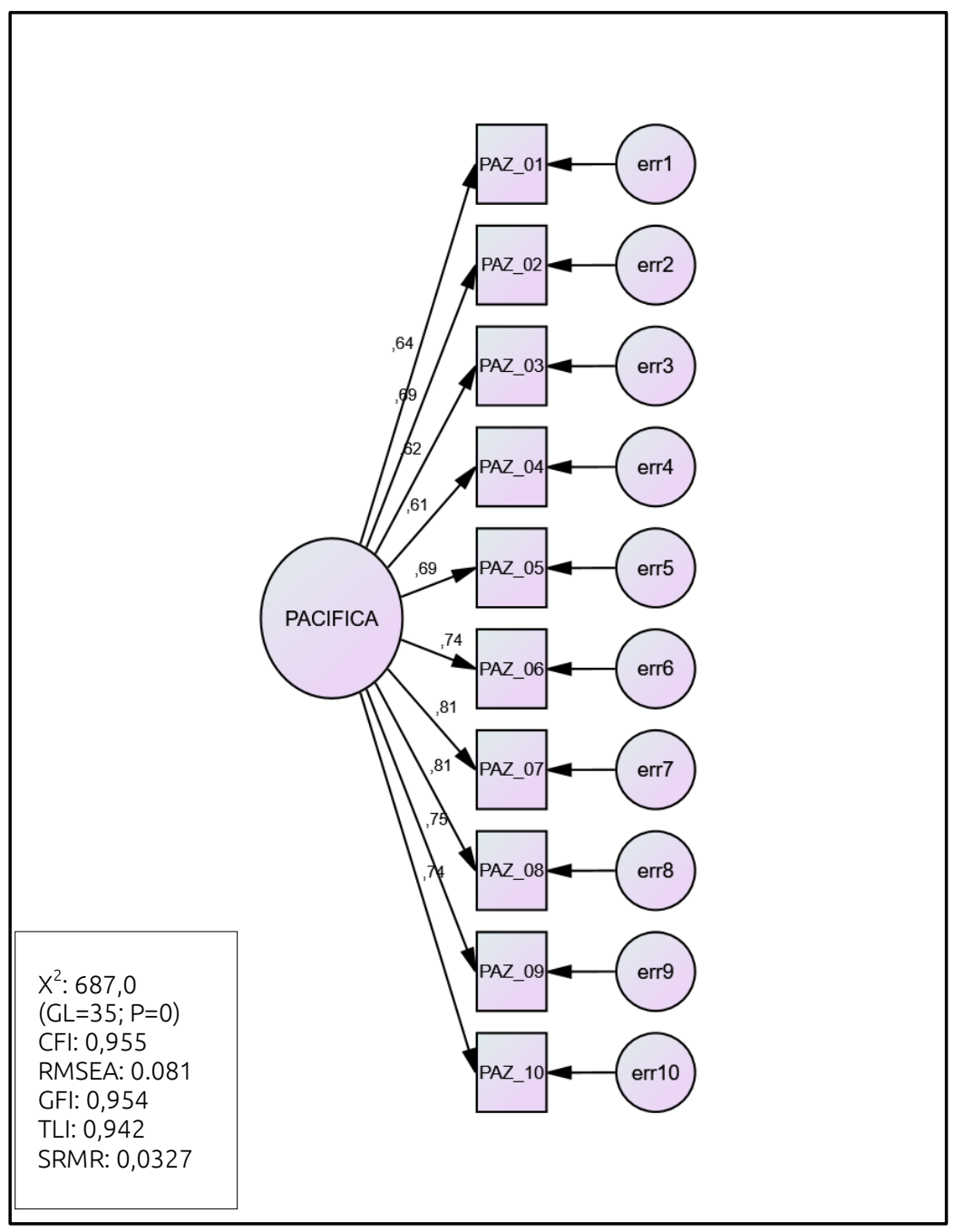

Figura 3. AFC para la dimensión Convivencia pacífica

Junto con los análisis confirmatorios, los resultados del estadístico de Cronbach indican muy buenos niveles de consistencia interna de cada una de las dimensiones (inclusión: $a=.88$; democracia: $a=.87$; paz: $a=.91)$.

\subsection{Análisis a la estructura completa del instrumento}

Debido a que las tres dimensiones analizadas de forma independiente forman parte de un mismo constructo, y dado que los autores (Caso et al., 2013; Chaparro et al., 2012, 2015) no llevaron a cabo un análisis que comprobara la pertinencia de las tres dimensiones a un solo constructo, se consideró pertinente realizar un AFE con este criterio, bajo el método de máxima verosimilitud con rotación Oblimin $(\mathrm{KMO}=.978 ;$ Bartlett $=43119.43 ; p<.00)$. Este análisis permite maximizar la diferencia entre factores para evidenciar soluciones factoriales consistentes. Los resultados sugieren una solución de 3 factores que en su conjunto explica el $42.4 \%$ de la varianza (requeridas 4 iteraciones), con un nivel de consistencia interna de .954, según alfa de Cronbach. Al mismo tiempo, se encontró un factor -al parecer subyacente- a las tres dimensiones del instrumento, lo que sugirió la presencia de un factor de nivel mayor, presuntamente Convivencia escolar. En cuanto a las correlaciones entre las dimensiones, podemos destacar que la dimensión Convivencia inclusiva presenta una correlación positiva y significativa con la dimensión Convivencia pacífica (.433). Sin embargo, la dimensión Convivencia democrática presenta una correlación significativa, pero negativa, con las dimensiones de Convivencia pacífica (-.787) y Convivencia inclusiva (- 
.410). Los niveles de correlación entre las dimensiones hacen evidente la existencia de una sola dimensión. Sin embargo, es importante considerar que dos de las correlaciones resultan negativas. Esto hace suponer que aunque evalúan el mismo constructo, lo hacen en direcciones distintas.

El AFC realizado para todo el instrumento sugirió un modelo factorial de segundo orden con índices de ajuste y cargas factoriales aceptables. De esta manera, se comprueba no sólo la estructura unifactorial de cada una de las dimensiones de primer orden, sino también la presencia de un factor subyacente de segundo orden, el cual -y dado el instrumento original- correspondería a convivencia escolar (figura 4).

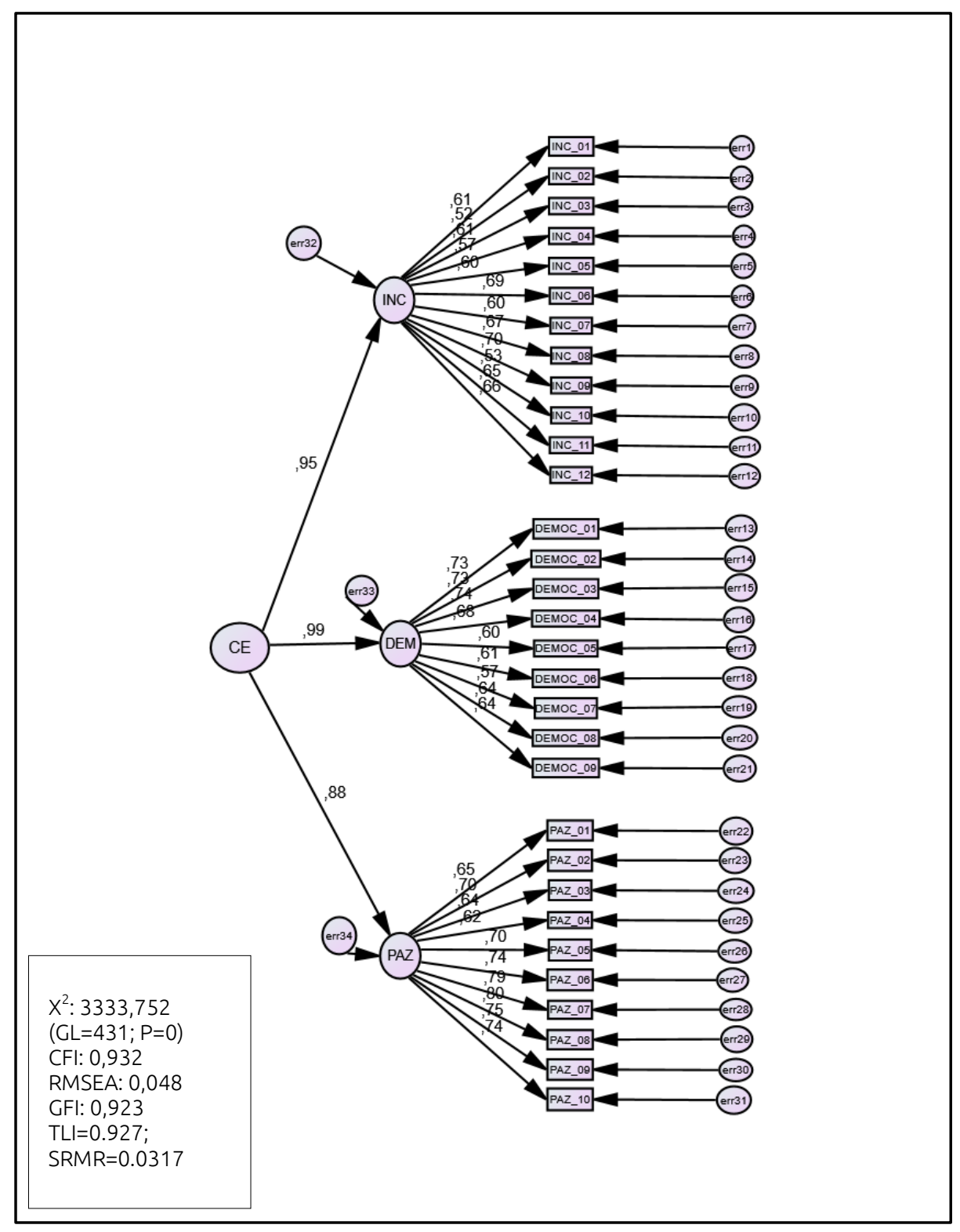

Figura 4. AfC para el instrumento completo

\section{Discusión}

Dado los pocos instrumentos disponibles para la evaluación de la convivencia escolar en Chile, especialmente desde el ámbito de las prácticas, la adaptación de un instrumento de evaluación ofrece 
esta posibilidad y permite analizar la convivencia en el país a través de estudios comparativos con México. El instrumento cuenta con dos características particulares: por un lado, busca medir prácticas de convivencia escolar, entendidas como acciones concretas que realizan los sujetos, especialmente los profesores, al interior de las escuelas (Chaparro et al., 2012, 2015; UNESCO, 2008); y por otro lado, el instrumento contempla indicadores claros y diferenciados para identificar prácticas de inclusión, de democracia y de paz.

En cuanto a la dimensión de Convivencia inclusiva, y mediante AFE, se configura la presencia de un factor que explica el $37.3 \%$ de la varianza con comunalidades mínimas y adecuadas entre reactivos. Esta información es confirmada por el AFC, mostrando que los indicadores de ajuste pertenecientes al modelo permiten confirmar la presencia de un factor que explica y subyace la dimensión de inclusión.

En relación con la dimensión de Convivencia democrática, y luego del AFE, los resultados indican la presencia de un factor que explica el $41.9 \%$ de la varianza. Si bien algunos ítems presentan comunalidades levemente bajas, no son suficientes para eliminar esos reactivos. Esta unidimensionalidad del factor es comprobada por el AFC, que arroja indicadores adecuados a la estructura factorial. Esta información confirma la robustez de la dimensión como un constructo único.

Los resultados de los AFC practicados a cada una de las tres dimensiones del instrumento mostraron adecuados índices de bondad de ajuste, lo que permitió confirmar la estructura del constructo de las tres dimensiones. Además, los niveles de consistencia interna resultaron en todos los casos con niveles bastante buenos.

En relación con el instrumento completo, y considerando las tres dimensiones a la vez, luego de un análisis factorial con método de máxima verosimilitud con rotación Oblimin, en los resultados, aunque si bien se agrupan en 3 factores que explican el $34.5 \%$ de la varianza, se observa la conformación de un factor mayor, el cual indica la presencia de un constructo mayor. Esta información se corroboró al analizar las correlaciones entre las dimensiones, pues los datos demuestran que son altas y que existe asociación entre ellas, aunque no sean correlaciones positivas. Además, haber encontrado ajuste en varios de los índices utilizados en el análisis factorial confirmatorio permite argumentar que la estructura de segundo orden puede funcionar mejor en la evaluación de la convivencia escolar. Sin embargo, la direccionalidad antes señalada no es esperable y podría estar dando cuenta de que, en los establecimientos chilenos, dadas las dificultades reportadas en la literatura respecto a la participación estudiantil como condición para la convivencia democrática (Ascorra, López y Urbina, 2016) y dado el carácter altamente disciplinar y autoritario bajo el cual se entiende la convivencia escolar (López, Díaz y Carrasco, 2015), una alta convivencia democrática, que necesariamente requiere ser participativa, puede ser entendida por los estudiantes como algo negativo para una convivencia inclusiva y pacífica. No obstante, esta hipótesis requiere de mayor exploración.

Considerando las tres dimensiones (Democrática, Inclusiva y Pacífica), los resultados del AFC de segundo orden dieron oportunidad de confirmar que las tres dimensiones comparten una dimensión subyacente: Convivencia escolar. Lo que confirma la apropiada estructura del instrumento en la evaluación del constructo desde las dimensiones planteadas por la UNESCO (2008), es decir, a través del instrumento se pueden evaluar las prácticas escolares que generan condiciones para la promoción de convivencia.

En conclusión, los resultados de la adaptación y validación del instrumento indican adecuadas características de validez y consistencia interna. Los resultados son similares al instrumento original (Caso et al., 2013), lo que constata, nuevamente, la calidad y rigurosidad del mismo. A su vez, la robustez de las características métricas permite, a futuro, la realización de estudios comparativos, pues por lo menos en las poblaciones mexicanas y chilenas estudiadas, se cuenta con un instrumento que se comporta de manera similar en ambos contextos culturales.

La importancia de contar con un instrumento que reporte las prácticas de una institución escolar radica principalmente en la posibilidad de reconocer gestiones formativas en los establecimientos, los cuales están ligados, según la literatura internacional, al mejoramiento de una escuela en convivencia escolar. Además, la relevancia de la presencia de dimensiones con énfasis en una convivencia inclusiva, 
democrática y pacífica es coherente con la gestión formativa o pedagógica señalada anteriormente, la que a su vez se relaciona con una escuela con proyección inclusiva. Por último, contar con instrumentos validados en distintas poblaciones latinoamericanas permite a la psicología avanzar hacia los estudios comparativos.

La principal limitación del estudio reside en el curso que sirvió de muestra para esta investigación; 8o. de enseñanza primaria o básica, pues la producción y el posterior análisis de información se inclinan por las percepciones de los participantes de un nivel en específico, dejando de lado edades y cursos de igual relevancia. El desafío frente a futuras investigaciones es comprobar la pertinencia del instrumento en otros niveles de enseñanza y en diferentes momentos de la escuela en un proceso de mejoramiento escolar.

\section{Referencias}

Abella, C., Rodríguez, D. y Pérez-Sindín (2010). Efectos del cambio de orden de variables y categorías de respuesta en los resultados de una encuesta política. Revista Metodología de Encuestas, 12, 7-27.

Ascorra, P., López, V. y Urbina, C. (2016). Participación estudiantil en escuelas chilenas con buena y mala convivencia escolar. Revista de Psicología, 25(2). doi:10.5354/0719-0581.2016.44686

Asún, R. (2006). Construcción de cuestionarios y escalas: el proceso de la producción de información cuantitativa. En M. Canales (Ed.), Metodologías de investigación social (pp. 63-113). Santiago de Chile: LOM.

Carifio, J. y Perla, R. (2007). Ten common misunderstandings, misconceptions, persistent myths and urban legends about likert scales and likert response formats and their antidotes. Journal of Social Sciences, 3(3), 106-116. Recuperado de http://thescipub.com/PDF/issp.2007.106.116.pdf

Carrasco, C. López, V. y Estay, C. (2012). Análisis crítico de la Ley de Violencia Escolar de Chile. Psicoperspectivas, 11(2), 31-55. Recuperado de http://www.psicoperspectivas.cl/index.php/psicoperspectivas/article/view/228

Caso, J., Díaz, C. y Chaparro, A. A. (2013). Aplicación de un procedimiento para la optimización de la medida de la convivencia escolar. Revista Iberoamericana de Evaluación Educativa, 6(2), 137-145.

Recuperado de http://www.rinace.net/riee/numeros/vol6-num2/art07.pdf

Chaparro, A. A., Caso, J., Díaz, C. y Urías, E. (2012). Instrumentos para el autodiagnóstico e intervención en escuelas basados en indicadores de convivencia democrática, inclusiva y no violenta. Reporte técnico 12-004. Universidad Autónoma de Baja California. Recuperado de

http://uee.uabc.mx/reportesTecnicos/12-004.php

Chaparro, A. A., Caso, J., Fierro, M. C., y Díaz, C. (2015). Desarrollo de un instrumento de evaluación basado en indicadores de convivencia escolar democrática, inclusiva y pacífica. Perfiles Educativos, 37(149), 20-41. Recuperado de http://www.iisue.unam.mx/perfiles//numeros/2015/149

Diario Oficial de la República de Chile (2015). Ley 20.845. Publicada en el Diario Oficial el 8 de junio de 2015.

Elijach, S. (2011). Violencia escolar en América Latina y el Caribe. Superficie y fondo. Panamá: UNICEF. Recuperado de https://www.unicef.org/lac/violencia escolar OK.pdf

Fierro, M. C. (2013). Convivencia inclusiva y democrática: Una perspectiva para gestionar la seguridad escolar. Sinéctica, 40, 01-18.

Fierro, M. C., Carbajal, P. y Martínez-Parente, R. (2010). Ojos que sí ven. Casos para reflexionar sobre la convivencia en la escuela. México: SM. 
Fierro, C., Tapia, G., Fortoul, B., Martínez-Parente, R., Macouzet, M. y Jiménez, M. (2013). Conversando sobre la convivencia en la escuela: Una Guía para el auto-diagnóstico de la convivencia escolar desde las perspectivas docentes. Revista Iberoamericana de Evaluación Educativa, 6(2), 103-124. Recuperado de http://www.rinace.net/riee/numeros/vol6-num2/art05.pdf

García, J. E. (2012). Conves: Evaluación de la convivencia escolar. Madrid: Tea Ediciones.

López, V., Díaz, H. y Carrasco, C. (2015). ¡Nosotros sí podemos! Aprendiendo a mejorar la convivencia escolar. Valparaíso: Marmor.

López, V., Carrasco, C., Morales, M., Ayala, A., López, J. y Karmy, M. (2011). Individualizando la violencia escolar: análisis de prácticas discursivas en una escuela municipal de la región de Valparaíso. Psykhe, 20(2), 7-23. doi:10.4067/S0718-22282011000200002

Lloret-Segura, S., Ferreres-Traver, A., Hernández-Baeza, A. y Tomás-Marco, I. (2014). El Análisis Factorial Exploratorio de los ítems: una guía práctica, revisada y actualizada. Anales de Psicología, 30(3), 1151-1169. doi:10.6018/analesps.30.3.199361

Mavrou, I. (2015). Análisis factorial exploratorio: cuestiones conceptuales y metodológicas. Revista Nebrija de Lingüistica Aplicada, 19. Recuperado de https://www.nebrija.com/revista-linguistica/files/articulos PDF/articulo 56583a3b9a601.pdf

Méndez, C. y Rondón, M. (2012). Introducción al análisis factorial exploratorio. Revista Colombiana de Psiquiatría, 41(1), 97-207. Recuperado de http://www.redalyc.org/articulo.oa?id=80624093014

Muñoz M. T., Lucero, B. A., Cornejo, C. A., Muñoz, P. A. y Araya, N. E. (2014). Convivencia y clima escolar en una comunidad educativa inclusiva de la Provincia de Talca, Chile. Revista Electrónica de Investigación Educativa, 16(2), 16-32. Recuperado de https://redie.uabc.mx/redie/article/view/497

Ortega, R. y Del Rey, D, R. (2002). Instrumentos para valorar la convivencia escolar. En R. Ortega, R. Del Rey, P. Gómez (Eds.), Estrategias educativas para la prevención de la violencia: mediación y diálogo. Recuperado de http://www.cruzrojajuventud.org/pls/portal30/docs/PAGE/SITE CRJ 2/AREA SEVICIOS/ ENLACES SERVICIOS/GUIA PREVENCION VIOLENCIA/CAPITULO6.PDF

Peralta, F. J. (2004). Estudio de los problemas de convivencia escolar en estudiantes de enseñanza secundaria (Tesis doctoral). Universidad de Málaga, España. Recuperado de

http://www.biblioteca.uma.es/bbldoc/tesisuma/16789222.pdf

Sandoval, M. (2014) Convivencia y clima escolar: claves de la gestión del conocimiento. última década, 22(41), 153-178. doi:10.4067/S0718-22362014000200007

UNESCO (2008), Convivencia democrática, inclusión y cultura de paz. Lecciones desde la práctica educativa innovadora en América Latina. Santiago de Chile: Pehuén Editores. 